\title{
On the Number of Tubes Touching a Sphere or a Tube
}

\author{
E.L. Starostin ${ }^{\dagger}$ \\ Max Planck Institute for Physics of Complex Systems, Nöthnitzer Str. 38, D-01187 \\ Dresden, Germany
}

October 18, 2005

\begin{abstract}
A problem is formulated about how many unit-radius tubes can touch a ball of given radius from the outside and from the inside. Upper bounds for the maximum numbers of contacts are obtained for both interior and exterior contacts. It is also shown that the maximum number of unit-radius tubes touching the same orthogonal cross-section of a particular tube of radius $P$ is $\left[\pi\left(\arcsin (P+1)^{-1}\right)^{-1}\right]$ and if the number of contacts takes on its maximum, then all tubes are locally aligned.
\end{abstract}

Keywords: packing, tube, bialy, kissing number, spherical codes, Tammes' problem

\section{Introduction}

The question of how many unit-radius perfect tubes can touch a given ball may be considered as a generalization of a question raised by Kuperberg, as attributed in [12] and [4], which in turn relates to the classical "kissing number" problem. The Kuperberg problem is to determine the maximum number of unit-radius infinite cylinders touching a unit-radius ball. To the best of the author's knowledge, the problem is still open: it is easy to find configurations with 6 cylinders and it was proved that 8 cylinders cannot be in contact with the ball.

An approach similar to that employed by Braß and Wenk [4] makes it also possible to obtain the estimate of the contact numbers for perfect tubes placed either outside or inside a sphere of arbitrary radius. The perfect tube is a basic notion in the ideal knot problem [20].

The paper contains three main parts, each devoted to a particular arrangement of bodies (perfect tubes and a sphere) in three-dimensional space. The first case is considered in Section 3 where the unit tubes are assumed to be in contact with a ball of arbitrary radius. A lemma is proved about an extremal property of a tube of a special shape (called a bialy). On the basis of this result, an upper bound estimate is obtained and formulated as Theorem 1. A connection of this construction with

$\dagger$ Current address: Department of Civil and Environmental Engineering, University College London, Gower Street, London WC1E 6BT, UK

Email: ucesest@ucl.ac.uk

(c) 2005 Kluwer Academic Publishers. Printed in the Netherlands. 
the Tammes problem [21] is established and discussed. This allows one to obtain additional estimates.

The methods of Section 3 are applied in the next section to tubes lying inside a sphere of greater radius and touching the spherical surface. The result is stated as Theorem 2. A duality property is established between the estimates for the exterior and interior of the sphere. Section 5 considers another type of problem when the central sphere is replaced by a tube of an arbitrary thickness radius. An upper bound for the number of tubes touching the same cross section of the central tube is formulated as Theorem 3. Possible applications of the results to various physical problems are discussed.

\section{Notation and preliminaries}

Let $\mathcal{C}$ be a space curve, piecewise of class $C^{2}: \mathcal{C}=\left\{\mathbf{r}: M \rightarrow \mathbb{R}^{3}\right\}$ with the arc length parameter $s$ such that $\mathbf{t}=\frac{d \mathbf{r}}{d s} \neq \mathbf{0}$ is a non-vanishing tangent vector. We shall be dealing normally with the cases when $M$ is either $\mathbb{R}$ for an infinite curve or $S^{1}$ for a closed one.

The global radius of curvature is defined in [10] as

$$
\rho_{G}(\mathbf{x})=\inf \left\{R_{c}(\mathbf{x}, \mathbf{y}, \mathbf{z}) \mid \mathbf{y}, \mathbf{z} \in \mathcal{C}, \mathbf{x} \neq \mathbf{y} \neq \mathbf{z} \neq \mathbf{x}\right\},
$$

where $R_{c}(\mathbf{x}, \mathbf{y}, \mathbf{z}) \geq 0$ is the radius of the smallest circle containing $\mathbf{x}, \mathbf{y}, \mathbf{z}$, i.e.

$$
R_{c}(\mathbf{x}, \mathbf{y}, \mathbf{z})=\frac{\|\mathbf{x}-\mathbf{y}\|}{2|\sin [\angle(\mathbf{x}-\mathbf{z}, \mathbf{y}-\mathbf{z})]|},
$$

if $\mathbf{x}, \mathbf{y}, \mathbf{z}$ are non-collinear; otherwise, for $\mathbf{x} \neq \mathbf{y} \neq \mathbf{z} \neq \mathbf{x}, R_{c}(\mathbf{x}, \mathbf{y}, \mathbf{z})$ is assigned the value of infinity. We restrict ourselves to curves with global radius of curvature at every point equal or greater than $\rho>0$.

We define a $\rho$-tube $\mathcal{T}$ based on the axis $\mathcal{C}: \mathcal{T}=\left\{\mathbf{x} \in \mathbb{R}^{3},\|\mathbf{x}-\mathbf{y}\|<\right.$ $\rho, \mathbf{y} \in \mathcal{C}\}$, and the closed $\rho$-tube $\overline{\mathcal{T}}=\left\{\mathbf{x} \in \mathbb{R}^{3},\|\mathbf{x}-\mathbf{y}\| \leq \rho, \mathbf{y} \in \mathcal{C}\right\}$. If the axis $\mathcal{C}$ is closed, then we call the tube a ring.

Since the global curvature does not exceed $\rho^{-1}$, the $\rho$-tube is embedded in $\mathbb{R}^{3}$, i.e. there exists a bijection from a straight cylinder (or a torus) onto the infinite tube (respectively, the ring). In this paper we fix the scale by setting $\rho=1$ and all but one of the tubes we shall be dealing with are 1-tubes. We shall call them either unit tubes or just tubes. The only exception is the $P$-tube considered in Section 5 .

An example of a ring that will be of particular interest to us, is a bialy $\mathcal{Y}=\mathcal{T}(\mathcal{C})$ with $\mathcal{C}$ a circle of unit radius [15]. In other words, a bialy is a torus with no hole.

Let $\mathcal{B}_{R}(\mathbf{z})=\left\{\mathbf{x} \in \mathbb{R}^{3},\|\mathbf{x}-\mathbf{z}\| \leq R\right\}$ be a ball of radius $R$ with its centre at $\mathbf{z}$. In particular, $\mathcal{B}_{P} \equiv \mathcal{B}_{P}(\mathbf{0})$ is the central ball of radius $P$. 
Any closed tube may be thought of as a union of balls: $\overline{\mathcal{T}}=\bigcup_{\mathbf{x} \in \mathcal{C}} \mathcal{B}_{\rho}(\mathbf{x})$.

The boundary of the ball is the sphere $\mathcal{S}_{R}(\mathbf{z})=\partial \mathcal{B}_{R}(\mathbf{z})$. We shall also use the notation $\mathcal{D}(\mathbf{r}(s)) \equiv \mathcal{D}(s)$ for a unit orthogonal cross-sectional disc of a tube: $\mathcal{D}(s)=\left\{\mathbf{r}(s)+\boldsymbol{\nu}(s), \boldsymbol{\nu}(s) \in \mathbb{R}^{3}, \boldsymbol{\nu}(s) \cdot \mathbf{t}(s)=0,\|\boldsymbol{\nu}(s)\| \leq\right.$ $1\}$.

\section{Tubes touching a ball from the outside}

Lemma 1. Let $\mathcal{B}_{P}$ be the central ball and $\mathcal{S}_{R}$ a central sphere of greater radius $R>P$. Let $\mathcal{T}$ be a tube with axis $\mathcal{C}=\left\{\mathbf{r}_{C}(s)\right\}$ touching $\mathcal{B}_{P}$ in point $Q=\overline{\mathcal{T}} \cap \mathcal{B}_{P}$. Let $A(\mathcal{C})$ be the intersection of the tube and the sphere: $A(\mathcal{C})=\overline{\mathcal{T}} \cap \mathcal{S}_{R}$. Then, for every $R$ in the range $P<R \leq P+2$, the area of $A$ reaches its minimum for $\mathcal{T}=\mathcal{Y}$.

Proof. In the following proof we consider only tubes which touch the $P$-ball at fixed $Q$. Since we seek a tube of minimal intersection area, we may restrict our consideration to tubes that only overlap with $\mathcal{S}_{R}$ on an interval of their axial arc length shorter than $\pi R$. We show first that for any such tube with non-planar axis $\mathcal{C}$, there exists a tube with planar axis having the same area of intersection with the $R$-ball.

Consider a generalized cone with its vertex in the origin and defined by the curve $\mathcal{C}$. The squared curvature of $\mathcal{C}$ can be decomposed as

$\kappa_{C}^{2}=\kappa_{C n}^{2}+\kappa_{C g}^{2}$, where $\kappa_{C n}$ is the normal curvature and $\kappa_{C g}$ is the geodesic curvature. The conical surface may be developed into a plane, so that $\mathcal{C}$ is mapped into $\mathcal{G}$, a planar curve with curvature $\kappa_{G}=\kappa_{C g}$. Note that since the global curvature of $\mathcal{C}$ does not exceed 1, this is certainly also true for $\mathcal{G}$.

The area of intersection of the tube $\mathcal{T}(\mathcal{C})$ with the $R$-sphere may be computed as

$$
\begin{aligned}
& \operatorname{Area}(A(\mathcal{C}))= \\
& =\lim _{\max \Delta s_{i} \rightarrow 0} \sum_{i} \operatorname{Area}\left(\left(\mathcal{S}_{R} \cap \mathcal{B}_{1}\left(\mathbf{r}_{C}\left(s_{i}+\Delta s_{i}\right)\right)\right) \backslash\left(\mathcal{S}_{R} \cap \mathcal{B}_{1}\left(\mathbf{r}_{C}\left(s_{i}\right)\right)\right)\right) .
\end{aligned}
$$

Here we used the fact that, for any $s_{k}<s_{l}<s_{m}$,

$$
\mathcal{B}_{1}\left(\mathbf{r}_{C}\left(s_{k}\right)\right) \cap\left[\mathcal{B}_{1}\left(\mathbf{r}_{C}\left(s_{m}\right)\right) \backslash \mathcal{B}_{1}\left(\mathbf{r}_{C}\left(s_{l}\right)\right)\right]=\emptyset,
$$

because of the constraint on the global curvature.

Developing the conical surface does not change the distances on the surface. In particular, this implies $r_{C}\left(s_{i}\right)=r_{G}\left(s_{i}\right), r_{C}\left(s_{i}+\Delta s_{i}\right)=$ $r_{G}\left(s_{i}+\Delta s_{i}\right)$ (here and below $\left.r \equiv\|\mathbf{r}\|\right)$. The relation between the balls Eq. (2) also remains valid: this follows from the fact that distances 
between the balls $\mathcal{B}_{1}$ may only increase. Consider an elementary area

$$
\begin{array}{r}
\Delta A_{C i}=\operatorname{Area}\left(\left(\mathcal{S}_{R} \cap \mathcal{B}_{1}\left(\mathbf{r}_{C}\left(s_{i}+\Delta s_{i}\right)\right)\right) \backslash\left(\mathcal{S}_{R} \cap \mathcal{B}_{1}\left(\mathbf{r}_{C}\left(s_{i}\right)\right)\right)\right)= \\
=\operatorname{Area}\left(\mathcal{S}_{R} \cap\left(\mathcal{B}_{1}\left(\mathbf{r}_{C}\left(s_{i}+\Delta s_{i}\right)\right) \backslash \mathcal{B}_{1}\left(\mathbf{r}_{C}\left(s_{i}\right)\right)\right)\right)= \\
=\operatorname{Area}\left(\mathcal{S}_{R} \cap \Delta \mathcal{B}\left(r_{C}\left(s_{i}\right), r_{C}\left(s_{i}+\Delta s_{i}\right), \Delta r_{C i}\right)\right)= \\
=\operatorname{Area}\left(\mathcal{S}_{R} \cap \Delta \mathcal{B}\left(r_{G}\left(s_{i}\right), r_{G}\left(s_{i}+\Delta s_{i}\right), \Delta r_{G i}\right)\right)+\mathcal{O}\left(\left(\Delta s_{i}\right)^{2}\right)= \\
=\Delta A_{G i}+\mathcal{O}\left(\left(\Delta s_{i}\right)^{2}\right),
\end{array}
$$

where $\Delta r_{C i} \equiv\left\|\mathbf{r}_{C}\left(s_{i}+\Delta s_{i}\right)-\mathbf{r}_{C}\left(s_{i}\right)\right\|=\Delta s_{i}+\mathcal{O}\left(\left(\Delta s_{i}\right)^{2}\right)$ and $\Delta r_{G i} \equiv$ $\left\|\mathbf{r}_{G}\left(s_{i}+\Delta s_{i}\right)-\mathbf{r}_{G}\left(s_{i}\right)\right\|=\Delta r_{C i}+\mathcal{O}\left(\left(\Delta s_{i}\right)^{2}\right)$. We can write

$$
\begin{array}{r}
\operatorname{Area}(A(\mathcal{C}))= \\
\lim _{\max \Delta s_{i} \rightarrow 0} \sum_{i} \operatorname{Area}\left(\left(\mathcal{S}_{R} \cap \mathcal{B}_{1}\left(\mathbf{r}_{G}\left(s_{i}+\Delta s_{i}\right)\right)\right) \backslash\left(\mathcal{S}_{R} \cap \mathcal{B}_{1}\left(\mathbf{r}_{G}\left(s_{i}\right)\right)\right)\right)= \\
=\operatorname{Area}(A(\mathcal{G})) .
\end{array}
$$

In other words, we have showed that the planar tube $\mathcal{G}$ has the same area of intersection with the $R$-sphere.

Now we may restrict ourselves to considering only planar axes for some interval $s \in[0, L]$. Let $s=0$ corresponds to a section that is in contact with the $P$-ball and $s=L$ to a section outside the $(P+2)$-ball. The function $r(s)$ starts at $r(0)=P+1$.

We consider first the case when the function $r(s)$ is monotonic. We are interested in the angle $\alpha$ measured from the tangent $\mathbf{t}(s)=\mathbf{r}^{\prime}(s)$ to the direction of $\mathbf{r}(s)$ (Fig. 1). If $\mathbf{r}(s)=\mathbf{r}_{0}(s)$ is an arc of a unit-radius circle with centre $A$ at distance $P+2$ from the origin, then it is easy to compute $\alpha=\alpha_{0}$ from the triangle $\triangle O A B$, applying the law of cosines: $(P+2)^{2}=1+r^{2}-2 r \cos \left(\frac{\pi}{2}+\alpha_{0}\right)$. Then $\alpha_{0}(r)=\arcsin \frac{(P+2)^{2}-1-r^{2}}{2 r}$. No other curve with constrained curvature can have a magnitude of $\alpha(r)$ less than $\alpha_{0}(r)$, because the curvature of $\mathbf{r}_{0}(s)$ is maximal.

Indeed, assume the contrary, i.e. that there exists a curve $\mathcal{E}$ which has an angle $\alpha(r)<\alpha_{0}(r)$ and the curvature of $\mathcal{E}$ does not exceed 1 . Consider a circle $\mathcal{C}_{1}$ with centre $A_{1}$ such that it has the common tangent with $\mathcal{E}$ in the point where both $\mathcal{C}_{1}$ and $\mathcal{E}$ intersect the circle of radius $r$ with centre $O$. The distance $O A_{1}$ is greater than $r$, but $O A_{1}<O A$ and the circle $\mathcal{C}_{1}$ intersects the $(P+1)$-circle while the curve $\mathcal{E}$ only touches the same $(P+1)$-circle, hence, at least a piece of $\mathcal{E}$ lies inside $\mathcal{C}_{1}$, but, according to Theorem 1 in Section 1.3 of Ref. [2], $\mathcal{E}$, having smaller curvature, must lie outside $\mathcal{C}_{1}$ (or they coincide). This contradiction proves that the angle $\alpha_{0}(r)$ is minimal.

Let $\mathcal{T}_{0}$ be the unit tube with $\mathbf{r}_{0}$ as its axis. Take some point on the axis $\mathbf{r}^{\star} \equiv \mathbf{r}_{0}\left(s^{\star}\right)$ and consider the ball $\mathcal{B}_{1}\left(\mathbf{r}^{\star}\right)$. Its intersection with the $R$-sphere is denoted by $Z^{\star}=\mathcal{S}_{R} \cap \mathcal{B}_{1}\left(\mathbf{r}^{\star}\right)$. Now take a small $\Delta r$, then 


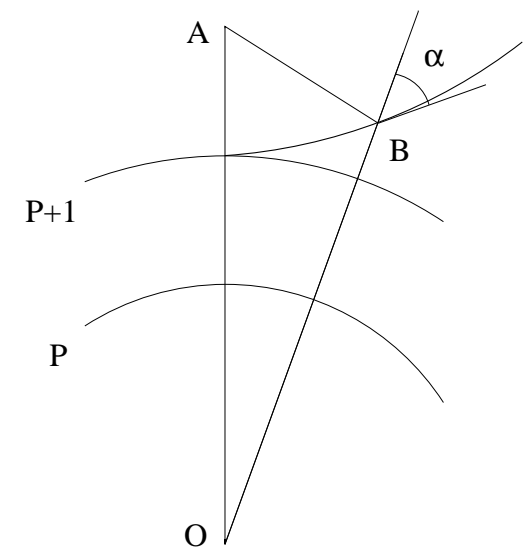

Figure 1. To the definition of the angle $\alpha$.

there exists a small $\Delta s$ such that $\left\|\mathbf{r}_{0}\left(s^{\star}+\Delta s\right)\right\|=\left\|\mathbf{r}^{\star}\right\|+\Delta r$. Define $Z_{d}=\mathcal{S}_{R} \cap \mathcal{B}_{1}\left(\mathbf{r}_{1}\right)$ for such $\mathbf{r}_{1}$ that $\left\|\mathbf{r}_{1}\right\|=\left\|\mathbf{r}^{\star}\right\|+\Delta r$. Compute the area of the difference

$$
\begin{array}{r}
\Delta A=\operatorname{Area}\left(Z_{d} \backslash Z^{\star}\right)=\operatorname{Area}\left(\mathcal{S}_{R} \cap\left(\mathcal{B}_{1}\left(\mathbf{r}_{1}\right) \backslash \mathcal{B}_{1}\left(\mathbf{r}^{\star}\right)\right)\right) \geq \\
\geq \operatorname{Area}\left(\mathcal{S}_{R} \cap\left(\mathcal{B}_{1}\left(\mathbf{r}_{0}\left(s^{\star}+\Delta s\right)\right) \backslash \mathcal{B}_{1}\left(\mathbf{r}^{\star}\right)\right)\right) .
\end{array}
$$

The inequality follows because the smaller $\alpha$ is, the smaller is the distance between two neighbouring balls sitting at levels $r^{\star}$ and $r_{1}$.

To compute the area of the entire intersection, we have to integrate $\Delta A$ over $r \in\left[P+1, r_{c r}\right]$. The last inequality above shows that the minimum is achieved for the unit circular axis, i.e. for a bialy.

The case of nonmonotonic $r(s)$ may be reduced to the monotonic case by cutting off the pieces of the tube axis where $r$ is less than its already achieved maximum, i.e. for $s \in K$, where $K=\{s: r(s)<$ $\left.\max _{0<\sigma<s} r(\sigma)\right\}$ The remaining pieces are to be shifted and glued together to make $r(s)$ continuous. It may happen that the resulting $r(s)$ is not smooth. Still, for this piecewise smooth curve, we again have $\alpha(r) \leq$ $\alpha_{0}(r)$. Denote by $\mathbf{r}_{j} \equiv \mathbf{r}\left(s_{j}\right)$ the points of the tangent discontinuities, $s_{j}<s_{j+1}, r_{j} \leq r_{j+1}$. The area of intersection of the initial tube $\mathcal{T}$ can be estimated as follows:

$$
\operatorname{Area}\left(\mathcal{S}_{R} \cap \mathcal{T}\right) \geq \sum_{j} \operatorname{Area}\left(\mathcal{S}_{R} \cap \mathcal{T}_{j}\right)-\sum_{j} \operatorname{Area}\left(\mathcal{S}_{R} \cap \mathcal{B}_{1}\left(\mathbf{r}_{j}\right)\right),
$$

where $\mathcal{T}_{j}$ is the unit tube based on $\mathbf{r}(s)$ for $\left[s_{j}, s_{j+1}\right]$.

Consider an interval where the shortened curve $\mathbf{r}$ is smooth. Suppose it lies between the levels $r_{j}$ and $r_{j+1}$. Applying the same argument as above, we can show that the axis of a tube that has the minimal 
intersection area with the $R$-sphere and that connects the levels $r_{j}$ and $r_{j+1}$ is a circular arc $\mathbf{r}_{0}$. Then,

$$
\sum_{j} \operatorname{Area}\left(\mathcal{S}_{R} \cap \mathcal{T}_{j}\right) \geq \sum_{j} \operatorname{Area}\left(\mathcal{S}_{R} \cap \mathcal{T}_{j}^{0}\right) .
$$

Inserting the last estimate into Eq. (3) yields

$$
\begin{array}{r}
\operatorname{Area}\left(\mathcal{S}_{R} \cap \mathcal{T}\right) \geq \sum_{j} \operatorname{Area}\left(\mathcal{S}_{R} \cap \mathcal{T}_{j}^{0}\right)-\sum_{j} \operatorname{Area}\left(\mathcal{S}_{R} \cap \mathcal{B}_{1}\left(\mathbf{r}_{j}\right)\right)= \\
=\operatorname{Area}\left(\mathcal{S}_{R} \cap \mathcal{T}^{0}\right),
\end{array}
$$

where $\mathcal{T}^{0}=\bigcup_{j} \mathcal{T}_{j}^{0}$ is (a piece of) the bialy.

Theorem 1. Let tubes $\mathcal{T}_{i}, i=1, \ldots, n$, be such that

1. $\mathcal{T}_{i} \cap \mathcal{B}_{P}=\emptyset$.

2. $\mathcal{T}_{i} \cap \mathcal{T}_{j}=\emptyset, i \neq j$.

3. $\overline{\mathcal{T}}_{i} \cap \mathcal{B}_{P}=Q_{i}, Q_{i}=\left\{\mathbf{q}_{i k} \in \mathbb{R}^{3}, k=1, \ldots, m_{i} \geq 1\right\}$ (q $\mathbf{q}_{i k}$ is the $k$-th contact point of the $i$-th tube with the central $P$-ball; the $i$-th tube has $m_{i}$ contact points).

4. $\forall i, k \exists \sigma_{i k}: \mathcal{D}\left(\sigma_{i k}\right) \cap \mathcal{B}_{P+2}=\emptyset, \mathcal{D}\left(s_{i k}\right) \cap \mathcal{B}_{P}=\mathbf{q}_{i k}, \infty<\sigma_{i 0}<s_{i 1}<$ $\sigma_{i 1}<s_{i 2}<\sigma_{i 2}<\ldots<s_{i k}<\sigma_{i k}<s_{i, k+1}<\ldots<\sigma_{i, m-1}<s_{i m}<$ $\sigma_{i m}<\infty$.

Then the total number of contacts is bounded: $\sum_{i=1}^{n} m_{i} \leq \tilde{N}(P)$, where the function $\tilde{N}(P)$ will be defined below. If $m_{i}=1, i=1, \ldots, n$, then the alternating condition 4 can be omitted and the theorem claims that $n \leq \tilde{N}(P)$.

Proof. First of all, we note that the intersections of tubes with any sphere concentric with the central ball are disjoint because the tubes are disjoint. We will consider further only spheres of radius $R$ such that $P \leq R \leq P+2$. As follows from the above lemma, the minimum area of intersection of the tube with a sphere of radius $R$ is achieved when the part of the tube inside the sphere takes the shape of a fragment of a bialy and the centre of the bialy is located at the (maximally possible) distance $P+2$ from the centre of the ball. For the sake of definiteness, let us fix the origin of the reference frame at the centre of the ball with the centre of the bialy lying on the $z$-axis and its circular axis being in the $x z$-plane. Then the bialy is described by the equation

$$
y^{2}+\left(\sqrt{x^{2}+(z-P-2)^{2}}-1\right)^{2}=1 .
$$


We are interested in its intersection with the sphere $\mathcal{S}_{R}$ given by

$$
x^{2}+y^{2}+z^{2}=R^{2} .
$$

The intersection domains are shown in Fig. 2 for $P=1, R=2$ (left) and $R=3$ (right).
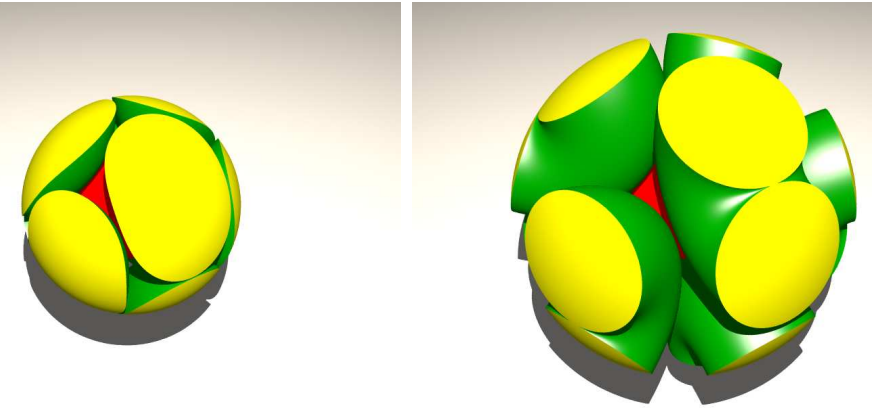

Figure 2. 9 unit bialies touching the unit ball and clipped with the sphere of radius $R=2$ (left) and $R=3$ (right).

The boundary of the overlapping region on the surface of the sphere satisfies the equation

$$
\left[R^{2}-2(P+2) z+(P+2)^{2}\right]^{2}=4\left[x^{2}+(z-P-2)^{2}\right]
$$

which is obtained after elimination of $y$ from Eqs. (4) and (5).

Let us introduce cylindrical coordinates by setting $x=\rho \cos \phi, y=$ $\rho \sin \phi, z=z$. Then Eq. (6) may be represented as a quadratic equation $4 U z^{2}-4 V z+W=0$ with coefficients depending on $P, R$ and $\phi$ :

$$
\begin{array}{r}
U=(P+1)(P+3)+\cos ^{2} \phi, \quad V=(P+2)\left[R^{2}+(P+2)^{2}-2\right], \\
W=\left[R^{2}+(P+2)^{2}\right]^{2}-4(P+2)^{2}-4 R^{2} \cos ^{2} \phi .
\end{array}
$$

Therefore, we have $z=z(\phi)=\frac{V \pm \sqrt{V^{2}-U W}}{2 U}$ (for $R \leq P+2$, only the sign "-" is meaningful).

Now we can compute the intersection area

$$
\begin{aligned}
S=\int_{0}^{2 \pi} \int_{0}^{\rho(\phi)} \frac{R}{\sqrt{R^{2}-\rho^{2}}} & \rho d \rho d \phi=R \int_{0}^{2 \pi}\left(R-\sqrt{R^{2}-\rho^{2}(\phi)}\right) d \phi= \\
& =4 R \int_{0}^{\pi / 2}(R-z(\phi)) d \phi=\frac{1}{2} S_{R}-4 R Z(P, R),
\end{aligned}
$$

where $S_{R}=4 \pi R^{2}$ is the entire area of the sphere $\mathcal{S}_{R}$ and $Z(P, R) \equiv$ $\int_{0}^{\pi / 2} z(\phi) d \phi$. The last integral may be expressed in terms of elliptic integrals. 
Let us consider the function $N=N(P, R)=\frac{S_{R}}{S}$,

$$
N^{-1}=\frac{1}{2}-\frac{1}{\pi R} Z(P, R) .
$$

We will show that, for fixed $P>\epsilon>0, N$ has a minimum in the interval $R \in(P, P+2)$ for some $R^{*}(P)$. To this end, we note first that for $R=P$ and $R=P+2$ both the function $Z(P, R)$ and its derivative with respect to $R$ may be expressed in elementary functions:

$$
\begin{array}{r}
\left.Z(P, R)\right|_{R=P}=\frac{\pi}{2} P, \\
\left.Z(P, R)\right|_{R=P+2}=\frac{\pi}{2}\left(2 \sqrt{(P+2)^{2}-1}-(P+2)\right), \\
\left.\frac{\partial Z(P, R)}{\partial R}\right|_{R=P}=\frac{\pi}{2}\left(1-\frac{2}{\sqrt{2(P+1)(P+2)}}\right)>0, \\
\left.\frac{\partial Z(P, R)}{\partial R}\right|_{R=P+2}=\frac{\pi}{2}\left(\frac{2(P+2)}{\sqrt{(P+2)^{2}-1}}-1\right)>0 .
\end{array}
$$

The derivative of $N^{-1}$

$$
\frac{\partial N^{-1}(P, R)}{\partial R}=\frac{1}{\pi R^{2}}\left(Z(P, R)-R \frac{\partial Z(P, R)}{\partial R}\right)
$$

has different signs at the ends of the interval:

$$
\left.\frac{\partial N^{-1}(P, R)}{\partial R}\right|_{R=P}=\frac{1}{P \sqrt{2(P+1)(P+2)}}>0
$$

and

$$
\left.\frac{\partial N^{-1}(P, R)}{\partial R}\right|_{R=R+2}=-\frac{1}{(P+2)^{2} \sqrt{(P+2)^{2}-1}}<0 .
$$

Applying the Weierstraß intermediate value theorem to the function $\frac{\partial N^{-1}(P, R)}{\partial R}$, we conclude that there exists $R^{*} \in(P, P+2)$ such that $\left.\frac{\partial N^{-1}(P, R)}{\partial R}\right|_{R=R^{*}}=0$, which implies that the function $N(P, R)$ has a local minimum on that interval. In order to find $R^{*}$, we solve numerically the equation

$$
Z(P, R)-R \frac{\partial Z(P, R)}{\partial R}=0 .
$$

Thus, for given $P$, the number of contacts cannot exceed $\tilde{N}(P)=$ $N\left(P, R^{*}(P)\right)$. A graph is shown in Fig. 3. The function $\tilde{N}(P)$ may be computed with arbitrary precision. Numerical computation suggests that for all fixed $P>0$ there is only one local minimum of $N(P, R)$. 


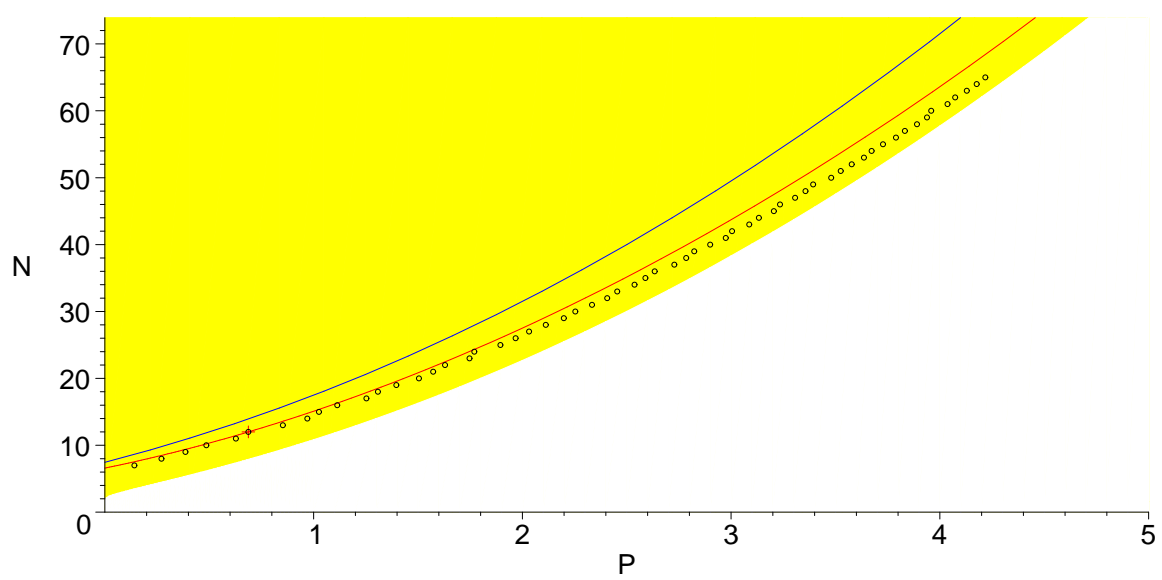

Figure 3. Shaded (yellow) region with boundary $\tilde{N}(P)$ corresponds to forbidden numbers of contacts of unit-radius tubes with the ball of radius $P$ from the outside, resp., with the sphere of radius $P+4$ from the inside. The upper (blue) curve represents the estimate Eq. (14). The lower (red) curve represents the improved upper bounds of Eqs. (15) and (16). Small circles correspond to the estimates obtained with spherical codes (see Remark 4). The cross marks the proved solution of the Tammes problem for 24 circles $(N=12)$.

However, the uniqueness property in no way affects the correctness of the estimate $\tilde{N}(P)$ because any $R$-sphere may be taken.

Remark 1. As can be seen from numerics, the function $Z(P, R)$ is convex in the range $(P, P+2)$ for any fixed $P>0$. Taking this to be a fact, we can thus consider the Legendre transform $G(P, Q)$ of $Z(P, R)$. Solving Eq. (13) is then equivalent to searching for a root $Q^{*}$ of $G$.

Remark 2. For the extreme value of radius $R=P+2$, Eq. (7) with Eq. (10) immediately implies $S=4 \pi R\left(R-\sqrt{R^{2}-1}\right)$ which is exactly the area of intersection of an infinite cylinder and an $R$-sphere centred on the axis of the cylinder. The cylinder crosses $\mathcal{S}_{R}$ orthogonally and the intersection consists of two spherical caps, each bounded by a unit-radius circle on the surface of the $R$-sphere. In the case of a unit bialy crossed with a sphere which passes through the bialy's centre, the intersection is also a pair of spherical caps of the same size (cf. Fig. 2 (right)). Indeed, the intersection must contain a pair of spherical caps, resulting from the intersection of the $R$-sphere with two disjoint unit balls centred at the axis of the bialy. Hence, the intersection area is bounded from below by $S$.

Inserting Eq. (10) into Eq. (8), we can obtain the estimate

$$
N^{-} \equiv N(P, P+2)=\frac{R}{R-\sqrt{R^{2}-1}}=\frac{P+2}{P+2-\sqrt{(P+1)(P+3)}},
$$


which is also shown in Fig. 3 as a solid line.

Remark 3. The estimate Eq. (14) can be improved. Assume that there are $N^{+}$contacts. Then the tubes must cross the $(P+2)$-sphere at least $2 N^{+}$times. The minimal area of every crossing is that of a unit spherical cap. Therefore the number $N^{+}$cannot exceed one half of the maximum number $n_{0}$ of free unit-radius circles packed on the sphere. The latter number corresponds to the solution of the Tammes problem [21] which is to find the configuration of a given number $n_{T}$ of points on the sphere that maximizes the minimum distance between any pair of points. The extremal configuration is called a spherical code [6]. A number of upper bounds are known for the Tammes problem. In Ref. [14], Fig. 1 presents a composite curve that corresponds to the two upper bounds proved by Robinson in $\S \S 9.1$ and 9.5 of Ref. [17]. In our terms, his expressions may be rewritten as follows:

$$
n_{0}<\frac{12\left(\pi R^{2}+\Sigma_{2}\right)}{4 \Sigma_{1}+\Sigma_{2}+\Sigma_{3}}, \quad \text { for } 12 \leq n_{0} \leq 24
$$

and

$$
n_{0}<\frac{6\left(\pi R^{2}-\Sigma_{2}+2 \Sigma_{3}\right)}{2 \Sigma_{1}+\Sigma_{3}}, \quad \text { for } n_{0} \geq 24,
$$

where $\Sigma_{1}$ is a spherical area of an equilateral triangle of side $2 \gamma$ (we denote its angles by $\left.\beta_{1}\right), \Sigma_{2}$ is a halved spherical area of a regular quadrangle of side $2 \gamma$ (we denote its angles by $\beta_{2}$ ) and $\Sigma_{3}$ is a spherical area of a triangle with two sides equal to $2 \gamma$ and included angle $2 \pi-4 \beta_{1}$. The formulas for the areas $\Sigma_{1}, \Sigma_{2}$ and $\Sigma_{3}$ are as follows:

$$
\begin{array}{r}
\Sigma_{1}=R^{2}\left(3 \beta_{1}-\pi\right), \quad \beta_{1}=\arccos \left(\frac{\cos 2 \gamma}{1+\cos 2 \gamma}\right), \\
\Sigma_{2}=R^{2}\left(2 \beta_{2}-\pi\right), \quad \beta_{2}=2 \arcsin \left(\frac{\sqrt{2}}{2 \cos \gamma}\right), \\
\Sigma_{3}=R^{2}\left(\pi-4 \beta_{1}-2 \beta_{3}\right), \quad \beta_{3}=\arctan \left(\frac{1+\cos 4 \beta_{1}}{\sin 4 \beta_{1} \cos 2 \gamma}\right) .
\end{array}
$$

The right-hand sides of Eqs. (15), (16) give upper bounds for the number of unit circles that can be packed on a sphere of radius $R=\csc \gamma$. For our problem, this implies that the number $N^{+}$of bialies that touch the ball of radius $P=R-2=\csc \gamma-2$ does not exceed $n_{0} / 2$. The curve corresponding to the bounds Eqs. (15), (16) is presented in Fig. 3.

Remark 4. The solutions to the Tammes problem are currently known with proofs only for all $n_{T} \leq 14$ and for $n_{T}=24$. A variety of existing numerical algorithms have produced presumably extremal configurations. The best solutions currently known are collected and updated on the web site [19] for all $n_{T} \leq 130$. 
Suppose we know the spherical code for a particular $n_{T}=2 m$, $m \in \mathbb{Z}^{+}$. The table [19] gives us the minimal value of the angle $2 \gamma$ between the centres of the spherical caps on the unit sphere. In other words, $n_{T}$ unit circles may be packed on the sphere of radius $R=\csc \gamma$. Therefore, not more than $N=m$ bialies can be in contact with the ball of radius $P=\csc \gamma-2$. Discrete points with coordinates $(P, N)$ are shown in Fig. 3 for $N \leq 65$. They were computed with the data from [19].

One can observe in Fig. 3 that the estimate based on spherical codes approaches $\tilde{N}$ as the radius $P$ increases. For smaller $P$, the spherical code estimates are getting closer to the curve of Eq. (14), while remaining better. Note also that two parts of the bound Eqs. (15) and (16) meet each other at a point for $N=12$, where both right-hand side expressions equal 24 which corresponds to the isolated proved solution to the Tammes problem [17].

Example 1. Let the central ball be of unit radius. With Eq. (8), we can compute $N(1,2) \approx 10.858914<11$. If we assume that there could be as many as 11 bialies touching the unit ball, then the total area of their overlappings with the sphere of radius 2 should exceed $S_{2}$, which is impossible.

Fig. 4 shows a symmetrical arrangement of 9 bialies touching the unit ball. Although gaps are seen between the bialies, it is not possible to add more bialies without moving those nine. Nevertheless, it is still not clear whether a configuration with 10 tubes exists or not.
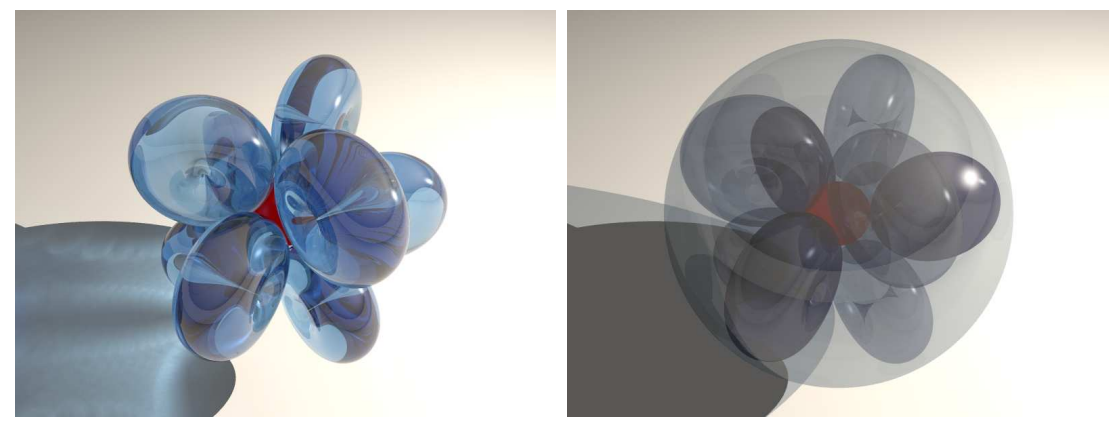

Figure 4. 9 unit bialies touch a unit ball. In the right figure they also touch a sphere of radius $P=5$ from the inside (see Example 2 in Sec. 4 ).

The constructions of six infinite cylinders touching a central ball (see Ref. [4]) does not contain a so-called rattler, i.e. a cylinder without contacts with other cylinders. This is not the case for the configuration presented in Fig. 4.

Another example of arrangement of tubes will be given at the end of the following section (Example 3). 


\section{Tubes touching the surface of a ball from the inside}

The approach developed above for the case when the tubes lie entirely outside the ball, may be adapted to the problem of tubes belonging to the interior of the ball. Of course, now we consider only rings. We start with modifying Lemma 1 and formulate

Lemma 2. Let $\mathcal{S}_{P}, P>2$ be a central sphere and $\mathcal{S}_{R}$ another central sphere of a smaller radius $R<P$. Let $\mathcal{T}$ be a tube inside $\mathcal{S}_{P}$ with axis $\mathcal{C}$ touching $\mathcal{S}_{P}$ in point $Q=\overline{\mathcal{T}} \cap \mathcal{S}_{P}$. Let $A(\mathcal{C})$ be the intersection of the tube and the $R$-sphere: $A(\mathcal{C})=\overline{\mathcal{T}} \cap \mathcal{S}_{R}$. Then for all $R$ such that $P-2 \leq R<P$ the area of $A$ reaches its minimum if $\mathcal{T}=\mathcal{Y}$.

The proof is similar to the proof of Lemma 1 .

Proceeding in the same way as in the exterior case we can compare the area of intersection of a bialy with an $R$-sphere and obtain the analogue of Theorem 1. However, it is possible to get a better upper bound if we consider how the remaining parts of the tubes can be packed inside a sphere of radius $P-2$.

Theorem 2. Let tubes $\mathcal{T}_{i}, i=1, \ldots, n$, be such that

1. $\mathcal{T}_{i} \cap \mathcal{B}_{P}=\mathcal{T}_{i}, P \geq 3$.

2. $\mathcal{T}_{i} \cap \mathcal{T}_{j}=\emptyset, i \neq j$.

3. $\overline{\mathcal{T}}_{i} \cap \mathcal{S}_{P}=Q_{i}, Q_{i}=\left\{\mathbf{q}_{i k} \in \mathbb{R}^{3}, k=1, \ldots, m_{i} \geq 1\right\}\left(\mathbf{q}_{i k}\right.$ is the $k$-th contact point of the $i$-th tube with the central $P$-sphere; the $i$-th tube has $m_{i}$ contact points).

4. $\forall i, k \exists \sigma_{i k}: \mathcal{D}\left(\sigma_{i k}\right) \cap \mathcal{B}_{P-2}=\mathcal{D}\left(\sigma_{i k}\right), \mathcal{D}\left(s_{i k}\right) \cap \mathcal{S}_{P}=\mathbf{q}_{i k}, 0 \leq s_{i 1}<$ $\sigma_{i 1}<s_{i 2}<\sigma_{i 2}<\ldots<s_{i k}<\sigma_{i k}<s_{i, k+1}<\ldots<\sigma_{i, m-1}<s_{i m}<$ $\sigma_{i m}<L_{i}$, where $L_{i}$ is the axis length of the $i$-th ring.

Then the total number of contacts is bounded: $\sum_{i=1}^{n} m_{i} \leq \tilde{N}_{i n}(P)$, where the function $\tilde{N}_{i n}(P)$ will be defined below.

Proof. Condition 4 means that between the contacts to the $P$-sphere each tube has to enter the $(P-2)$-ball so that there should exist a crosssectional disc belonging to this small ball. This is always possible for $P \geq 3$. Now we take the $i$-th ring and we shall normally omit the index $i$ in the sequel for simplicity.

Let $\mathbf{r}(s), s \in M, s_{i k}<s<s_{i, k+1}$ be the axis of the piece(s) of the $i$-th ring inside the $(P-2)$-ball. Then $r^{\star} \equiv \min _{s \in M} r(s) \leq P-3$, because otherwise no cross-section would be immersed in the $(P-2)$ ball. The plane of the section $\mathcal{D}\left(\mathbf{r}^{\star}\right)$ passes through the origin and the 
tube touches the surface of the ball of radius $\rho \leq|P-4|$ at a point that belongs to $\mathcal{D}\left(\mathbf{r}^{\star}\right)$. Note that the $\rho$-ball lies inside the closed tube for $3 \leq P<4$. Then, since all the tubes are disjoint, it follows immediately that only one ring has room inside the $(P-2)$-ball, i.e. $\tilde{N}_{i n}(P)=1$, $P \in[3,4)$.

Now let $P \geq 4$. We consider a sphere of radius $R \leq P-2$ and a piece of ring inside this sphere. We are interested in determining the minimal normalized area of the intersection of the ring with the $R$ sphere. If $r^{\star} \geq R-1$ for this piece of the ring, then we may apply Lemma 1 and claim that the minimal area is reached when the piece is part of the bialy. If the ring sinks deeper into the $(P-2)$-ball, i.e. if $r^{\star}<R-1$, then nothing prevents the ring from crossing the $R$ sphere orthogonally and the intersection is simply a pair of spherical caps bounded by unit circles.

We need not consider tubes that enter the $(P-4)$-ball. Indeed, let $N^{-1}\left(P-4, R_{0}\right)$ be the normalized intersection area for a bialy that is in contact with the $(P-4)$-ball. The bialy is crossed with the sphere of some radius $R_{0} \in(P-4, P-2)$. Now consider another bialy that touches a ball of smaller radius $P_{1}<P-4$. It is always possible to choose the radius of the crossing sphere $R_{1}=R_{0} P_{1} /(P-4)$. Then, $N^{-1}\left(P_{1}, R_{1}\right) \geq N^{-1}\left(P-4, R_{0}\right)$, because we have proportionally deflated both the contacting ball and the crossing sphere, but left the thickness radius of the bialy unchanged. Since we are seeking a minimum of $N^{-1}$ among all possible configurations of the ring, it is sufficient to consider only the bialies with centres at $\mathcal{S}_{P-2}$. These bialies touch the $(P-4)$-ball. Then the intersection area is given by Eq. $(7)$, but we should formally substitute $P \rightarrow P-4$ in the expressions for the coefficients $U, V, W$.

Now we can define the function $N_{i n}(P, R) \equiv N(P-4, R)$ where $N$ is given by Eq. (8). For fixed $P \geq 4, N_{i n}$ has a minimum in the interval $R \in(P-4, P-2): \tilde{N}_{i n}(P)=\min _{R \in(P-4, P-2)} N_{i n}(P, R)$, which is an upper bound to the number of contacts of the rings with the $P$-sphere. The graph and the estimate in the interior case are essentially the same as in the exterior one, only shifted along the $P$-axis by 4 (Fig. 3). Thus, we establish the duality property of the estimate: the same value serves both to bound the number of unit tubes touching the surface of the small $P$-ball from the outside and of the larger $(P+4)$-ball from the inside.

Remark 5. The alternating condition 4 in the formulation of Theorem 2 cannot be omitted as can be seen from the following example. Consider two bialies arranged so that they touch each other along a circle. They can be packaged into a sphere of radius $P=\sqrt{2}+1<3$. 
To reduce the contacts to the sphere to one point for each bialy, one can increase $P$ by an arbitrary small value and move the bialies accordingly. Clearly, condition 4 is violated for such a configuration. At the same time, we have $m_{1}+m_{2}=1+1=2=n$ which exceeds the above upper bound $\tilde{N}_{i n}(P)=1$, for $P$ up to 4 . Note also that in the analogue of Theorem 1 mentioned above (before formulation of Theorem 2) condition 4 can be left out exactly as in Theorem 1 .

Example 2. On the right of Fig. 4 one can see a symmetrical arrangement of 9 bialies touching a sphere of radius $P=5$ from the inside. This is the same configuration as that considered in Example 1 in Sec. 3. Similarly, our estimate excludes an arrangement of 11 tubes, but not 10 .

Example 3. An approximation of the ideal Borromean rings was proposed in [5] (Fig. 5). The whole structure was composed of three identical rings each having a plane centreline made of four circular arcs. There is room in the central part of the structure for a ball of radius $\sqrt{7}-2$ and each ring touches the ball twice.

The rings may be placed inside a sphere of radius $\sqrt{7}+2$ so that each ring has two contact points with the sphere. Thus, in both cases, the total number of contacts is 6 , while we have $7<\tilde{N}(\sqrt{7}-2)=$ $\tilde{N}_{i n}(\sqrt{7}+2)<8$ for the upper bound function. So, it does not rule out the existence of another structure with 7 contact points for the same radii of the inner ball and the outer sphere, but 8 points are forbidden.
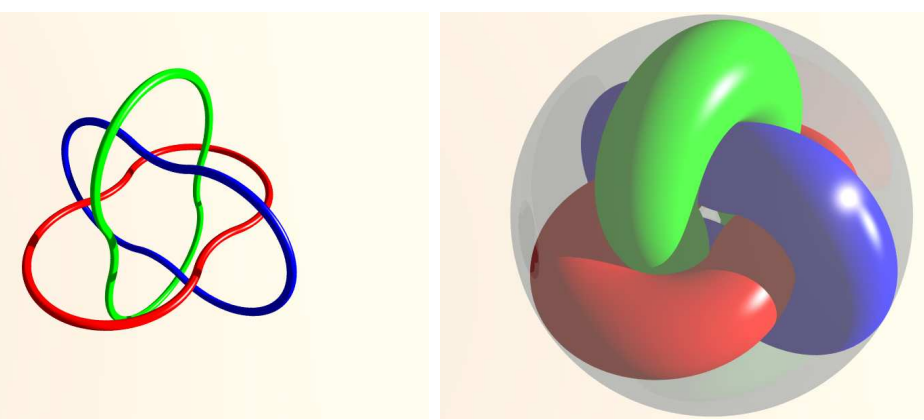

Figure 5. Borromean rings made of pieces of tubes with circular axes. In the right figure the same rings have unit radius of thickness and touch a sphere of radius $P=\sqrt{7}+2$.

\section{On the number of tubes touching a given tube}

In this section we prove a theorem related to the situation when instead of a ball there is a tube in the centre. 
Theorem 3. Let $\mathcal{T}_{0}$ be a tube of radius $P$ and $\mathcal{D}_{0}$ be its arbitrary section. Let unit tubes $\mathcal{T}_{i}, i=1, \ldots, n$, be such that

1. $\mathcal{T}_{\lambda} \cap \mathcal{T}_{\mu}=\emptyset, \lambda \neq \mu ; \lambda, \mu=0, \ldots, n$, and

2. $\overline{\mathcal{T}}_{i} \cap \mathcal{D}_{0}=Q_{i}, Q_{i}=\left\{\mathbf{q}_{i k} \in \mathbb{R}^{3}, k=1, \ldots, m_{i} \geq 1\right\}\left(\mathbf{q}_{i k}\right.$ is the $k$ th contact point of the $i$-th tube, $i=1, \ldots, n)$. It belongs to the cross-sectional disc $\mathcal{D}_{i k}$ with normal $\mathbf{n}_{i k}$.

Then the number of touching unit tubes $n$ does not exceed $\pi \arcsin (P+$ $\left.1)^{-1}\right)^{-1}$. If each unit tube has exactly one contact with the central one $\left(m_{i}=1\right)$ and $P=\csc \frac{\pi}{n}-1$, then all $n+1 \operatorname{discs} \mathcal{D}_{0}$ and $\mathcal{D}_{i 1}$ lie in the same plane.

Proof. Without loss of generality, we place the centre of the disc $\mathcal{D}_{0}$ into the origin and we call it the central disc. Let $\mathbf{r}_{i k}$ be the centres of the other discs $\mathcal{D}_{i k},\left\|\mathbf{r}_{i k}\right\|=P+1, i=1, \ldots, n, k=1, \ldots, m_{i}$. The tangent to the axis of the central tube in the origin $\mathbf{n}_{0}$ is the normal to the central disc and it is orthogonal to all $\mathbf{r}_{i k}$, i.e. the centres of all $n+1$ discs lie in the same plane $\mathcal{P}_{0}$ which also contains $\mathcal{D}_{0}$. Since $\mathcal{D}_{0} \cap \mathcal{D}_{i k}=\left\{\mathbf{q}_{i k}\right\} \neq \emptyset$, we may write $\mathcal{B}_{P} \cap \mathcal{B}_{1}\left(\mathbf{r}_{i k}\right)=\left\{\mathbf{q}_{i k}\right\} \neq \emptyset$, i.e. the balls $\mathcal{B}_{P}$ and $\mathcal{B}_{1}\left(\mathbf{r}_{i k}\right)$ have no common interior points. The last is also true for the balls $\mathcal{B}_{1}\left(\mathbf{r}_{i k}\right)$ and $\mathcal{B}_{1}\left(\mathbf{r}_{j l}\right)(i \neq j)$, because, for any $i$, the interior of $\mathcal{B}_{1}\left(\mathbf{r}_{i k}\right)$ belongs to $\mathcal{T}_{i}$ and the tubes do not overlap.

Clearly, the number $n$ of unit tubes is not greater than the number of unit balls touching the central ball, all having their centres coplanar. But that number, in turn, cannot exceed the kissing number in $\mathbb{R}^{2}$, which is $\left[\pi\left(\arcsin (P+1)^{-1}\right)^{-1}\right]$ for unit circles contacting the common central circle of radius $P$ (see Fig. 6 ). This proves the first claim of the theorem.

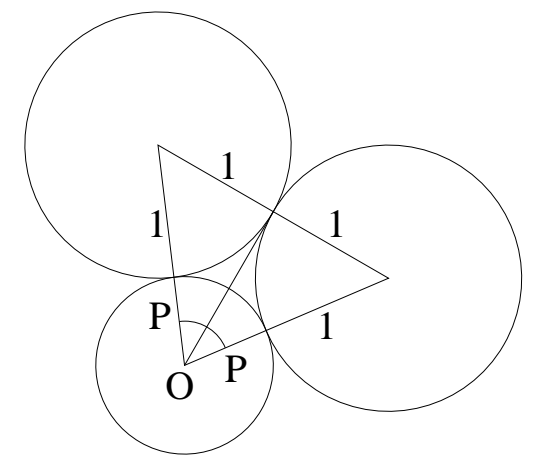

Figure 6. Sections of the balls by the plane $\mathcal{P}_{0}$ orthogonal to the axis of the central tube.

Now suppose that all the balls belong to different tubes $\left(m_{i}=1\right)$ and that $P=\csc \frac{\pi}{n}-1$. We have $n$ coplanar points $\mathbf{r}_{i 1} \in \mathcal{P}_{0}$, each at 
distance $P+1$ from the origin and the distance between each pair of them is at least 2 . This is only possible if they are vertices of a regular $n$-gon and they may be renumbered so that $\left\|\Delta \mathbf{r}_{j}\right\|=2, \Delta \mathbf{r}_{j} \equiv \mathbf{r}_{j+1}-\mathbf{r}_{j}$, $j=1, \ldots, n$ (we identify $\mathbf{r}_{n+1} \equiv \mathbf{r}_{1}$ ). This means that the piece of tube corresponding to $\mathbf{r}_{j}$ is in contact with both the central tube and two other tubes, $\mathcal{T}_{j \pm 1}$. The tangent to the axis of the $j$-th tube at $\mathbf{r}_{j}$ is $\mathbf{n}_{j}$, the normal to $\mathcal{D}_{j}$. Then $\mathbf{n}_{j} \cdot \mathbf{r}_{j}=0$ and $\mathbf{n}_{j} \cdot \Delta \mathbf{r}_{j}=0$, which implies that $\mathbf{n}_{j}$ is normal to the plane $\mathcal{P}_{0}$, i.e. all $\mathcal{D}_{j} \in \mathcal{P}_{0}$.

Example 4. If the central tube also has the same unit radius of thickness, then $n \leq 6$.

Remark 6 . To avoid situations when one tube may have an arbitrary number of contacts with the central disc, e.g. by having its axis coplanar with the disc, an additional condition can be included requiring that the unit tubes should be sufficiently "straight", i.e. that the radius of curvature should be larger then $P+1$.

Corollary. Let the maximum allowed number of tubes be in continuous contact with the central tube whose axis is $\mathbf{r}_{0}(s), s$ being the arc length parameter. Then the second part of Theorem 3 implies that the vector field $\Delta \mathbf{r}_{j 0}(s) \equiv \mathbf{r}_{j}(s)-\mathbf{r}_{0}(s)$ is relatively parallel [3].

Proof. Since the vector $\Delta \mathbf{r}_{j 0}(s)$ does not vary in magnitude, we may write

$$
\frac{d \mathbf{r}_{j}(s)}{d s}=\frac{d \mathbf{r}_{0}(s)}{d s}+\boldsymbol{\omega} \times \Delta \mathbf{r}_{j 0}(s),
$$

where $\boldsymbol{\omega}$ is the angular velocity of rotation of the orthogonal frame $\left\{\mathbf{n}_{0}(s), \Delta \mathbf{r}_{j 0}(s), \mathbf{n}_{0}(s) \times \Delta \mathbf{r}_{j 0}(s)\right\}$ as $s$ varies. Computing the crossproduct of Eq. (17) with $\mathbf{n}_{0}(s)$ yields $\boldsymbol{\omega} \cdot \mathbf{n}_{0}(s)=0$, because the tangent $\frac{d \mathbf{r}_{j}(s)}{d s}$ is parallel to $\mathbf{n}_{0}(s)$. In other words, the twist rate of the vector field $\Delta \mathbf{r}_{j 0}(s)$ is zero. Representing the vector $\boldsymbol{\omega}$ as a sum of two components $\boldsymbol{\omega}=\omega_{1} \Delta \mathbf{r}_{j 0}(s)+\omega_{2} \mathbf{n}_{0}(s) \times \Delta \mathbf{r}_{j 0}(s)$, we find

$$
\frac{d \Delta \mathbf{r}_{j 0}(s)}{d s}=-\omega_{2} \Delta \mathbf{r}_{j 0}^{2}(s) \mathbf{n}_{0}(s),
$$

i.e. the derivative of the vector $\Delta \mathbf{r}_{j 0}(s)$ remains tangential to $\mathbf{r}_{0}(s)$.

Example 5. Suppose that the axis $\mathbf{r}_{0}(s)$ is smoothly closed. Let the other tubes be closed, too. We denote by $\mathcal{L} k_{j}$ the linking number of $\mathbf{r}_{0}(s)$ and $\mathbf{r}_{j}(s)$. Then the writhing number of each of the curves $\mathbf{r}_{0}(s)$ and $\mathbf{r}_{j}(s)$ equals $\mathcal{L} k_{j}$ and, consequently, it is integer. This follows from the famous Călugăreanu-White-Fuller theorem $\mathcal{L} k=\mathcal{T} w+\mathcal{W} r$ [9], because the twisting number vanishes.

The property of the relative parallelism and an integer writhe (for closed configurations) may be applied to describe toroidal conformations produced as a result of DNA condensation, characterized by hexagonal lattice packing $[16,13]$. In particular, a dense packing of 
crystallized DNA exists in virus capsids [8]. The above consideration implies that the toroids as a whole cannot take an arbitrary shape, once we assume the existence of 6 adjacent strands everywhere. Similarly, nematic phases of long polymers should obey the same rules. Another possible application could be the theory of multistrand cables and wire ropes [7].

\section{Concluding remarks}

1. Condition 4 in Theorem 1 about the existence of a section outside the $(P+2)$-ball (and its analogue in Theorem 2$)$ is natural because it allows another piece of tube to come into contact, thus excluding a situation where a tube warps nearly around the ball and can have an arbitrary large number of contacts. Introduction of the above condition makes meaningful the question about how many pieces of tubes can come into contact with the ball. However, condition 4 plays no role in the upper bound estimates of the number of contacting tubes.

2. As mentioned by Gray [11], the volume of a tube does not depend on its curvature. This is true if the thickness radius does not exceed the (global) radius of curvature. A generalization to tubes of varying thickness is possible. An example of this is the strip-like intersection of a tube and a sphere (cf. Lemma 1).

3. The paper [18] deals with equilibria of a tubular polymer chain attracted to a spherical organizing centre. In particular, an upper bound for the number of contacts is estimated to be of order $P^{3 / 2}$ (in our notation). Fig. 3 suggests that this estimate does not fit the behaviour of $\tilde{N}$. This can be justified by taking into account the limited flexibility of the polymer (DNA) which may imply an upper bound constraint on the local curvature of the axis. Indeed, for the extreme case of absolutely rigid straight cylinders an upper bound of order $P^{3 / 2}$ may be obtained [4]. The approach employed above may be readily extended to tubes of limited flexibility.

4. Recently, a tube model has been proposed to better understand the geometry of protein folding [1]. In view of this approach, the estimates for the contact numbers may be useful when applied to globular proteins to count exposed fragments of the amino acids chain. One can also suggest that the above estimates may serve as steric constraints for the validation of computed secondary structures of RNA. 


\section{References}

1. Banavar, J. R. and A. Maritan: 2003, 'Colloquium: Geometrical approach to protein folding: a tube picture'. Reviews of Modern Physics 75(1), 23-34.

2. Berger, M.: 2003, A Panoramic View of Riemannian Geometry. Springer.

3. Bishop, R. L.: 1975, 'There is more than one way to frame a curve'. Amer. Math. Monthly 82(3), 246-251.

4. Braß, P. and C. Wenk: 2000, 'On the number of cylinders touching a ball'. Geometriae Dedicata 81, 281-284.

5. Cantarella, J., R. B. Kusner, and J. M. Sullivan: 2002, 'On the minimum ropelength of knots and links'. Inventiones Mathematicae 150(2), 257-286.

6. Conway, J. H. and N. J. A. Sloane: 1999, Sphere Packings, Lattices and Groups, Vol. 290 of Grundlehren der mathematischen Wissenschaften. A Series of Comprehensive Studies in Mathematics. New York: Springer, 3 edition.

7. Costello, G. A.: 1997, Theory of Wire Rope, Mechanical Engineering Series. Springer, 2 edition.

8. Earnshaw, W. C. and S. C. Harrison: 1977, 'DNA arrangement in isometric phage heads'. Nature 268, 598-602.

9. Fuller, F. B.: 1971, 'The writhing number of a space curve'. Proc. Natl. Acad. Sci. USA 68(4), 815-819.

10. Gonzalez, O. and J. H. Maddocks: 1999, 'Global curvature, thickness, and the ideal shapes of knots'. Proc. Natl. Acad. Sci. USA 96, 4769-4773.

11. Gray, A.: 2004, Tubes, Vol. 221 of Progress in Mathematics. Basel-BostonBerlin: Birkhäuser, 2 edition.

12. Heppes, A. and L. Szabó: 1991, 'On the number of cylinders touching a ball'. Geometriae Dedicata 40, 111-116.

13. Hud, N. V. and K. H. Downing: 2001, 'Cryoelectron microscopy of lambda phage DNA condensates in vitreous ice: The fine structure of DNA toroids'. Proc. Natl. Acad. Sci. USA 98(26), 14925-14930.

14. Kottwitz, D. A.: 1991, 'The densest packing of equal circles on a sphere'. Acta Cryst. A 47, 158-165.

15. Kusner, R.: 2002, In: Physical Knots: Knotting, Linking, and Folding Geometric Objects in $\mathbb{R}^{3}$, eds. J. A. Calvo, K. C. Millett, E. J. Rawdon, Vol. 304 of Contemporary Mathematics, Chapt. On thickness and packing density for knots and links. American Mathematical Society.

16. Livolant, F., A. M. Levelut, J. Doucet, and J. P. Benoit: 1989, 'The highly concentrated liquid-crystalline phase of DNA is columnar hexagonal'. Nature 339, 724-726.

17. Robinson, R. M.: 1961, 'Arrangement of 24 points on a sphere'. Mathematische Annalen 144, 17-48.

18. Schiessel, H., J. Rudnick, R. Bruinsma, and W. M. Gelbart: 2000, 'Organized condensation of worm-like chains'. Europhysics Letters 51(2), 237-243.

19. Sloane, N. J. A., R. H. Hardin, W. D. Smith, et al., 'Tables of Spherical Codes'. Published electronically at www.research.att.com/ njas/packings/.

20. Stasiak, A., V. Katritch, and L. H. Kauffman (eds.): 1998, Ideal Knots, Vol. 19 of Series on Knots and Everything. World Scientific.

21. Tammes, P. M. L.: 1930, 'On the origin of number and arrangement of the places of exit on the surface of pollen-grains'. Recueil des Travaux Botaniques Néerlandais 27, 1-84. 